\title{
GENOMIQUE ET LIPIDES Génomique et métabolisme des lipides des plantes
}

\section{Genomics and Lipids Genomics and plant lipid metabolism}

Oléagineux, Corps Gras, Lipides. Volume 9, Numéro 2, 130-4, Mars - Juin 2002, Dossier : Génomique et filière oléagineuse - Journées Chevreul de l' AFECG, Paris, 21-22 novembre 2001

Auteur(s) : Michel DELSENY, Lionel VERDOUCQ, Sylvie MAISONNEUVE, Thomas ROSCOE, Laboratoire génome et développement des plantes, UMR 5096, CNRS-IRD-UP Université de Perpignan, 66860 Perpignan Cedex, France.

Author(s) : Michel DELSENY, Lionel VERDOUCQ, Sylvie MAISONNEUVE, Thomas ROSCOE

Résumé : Il existe dans les bases de données publiques une énorme quantité de séquences d'ADN dérivées de plantes, et notamment la séquence complète du génome d'Arabidopsis thaliana, une plante modèle pour les oléagineux, proche parente du colza. Ces données constituent une ressource importante non seulement pour la compréhension de métabolisme lipidique et de sa régulation, mais aussi pour la sélection et le développement de variétés nouvelles d'oléagineux produisant davantage d'huiles ou des huiles de composition nouvelle. Cette abondance de séquences peut être exploitée, en utilisant les recherches d'homologies, pour identifier les gènes, pour obtenir des informations sur leur fonction, comme pour repérer des gènes candidats codant des fonctions nouvelles. L'analyse de ces bases de données a révélé que la majeure partie des gènes codant des enzymes impliquées dans le métabolisme lipidique appartient à des petites familles multigéniques, reflétant la diversification des fonctions des isoformes. Une analyse du catalogue des ADNc séquencés en aveugle reflète les niveaux d'expression des différents gènes et fournit un aperçu des régulations des flux au travers des voies métaboliques conduisant à la biosynthèse des lipides de réserve. La disponibilité de mutants et de lignées transgéniques d'Arabidopsis et le développement de puces à ADN qui permettent l'analyse simultanée de plusieurs milliers de gènes conduiront à une meilleure compréhension des facteurs qui régulent le métabolisme des huiles dans les graines. Une telle connaissance facilitera la manipulation de la composition des huiles et des quantités produites dans les graines.

Summary : An enormous quantity of DNA sequence data derived from plants can be found in public databases, including the entire genomic sequence of Arabidopsis thaliana, a model for oilseeds, closely related to Brassica napus. This data constitutes an important resource not only for the understanding of lipid metabolism and its regulation but also for the selection and development of new varieties of oilseeds with enhanced production of storage lipids possessing novel fatty acid compositions. This abundance of sequence data may be exploited using homology searches to identify genes and provide insight as to their probable function as well as reveal the presence of candidate genes encoding proteins uncharacterised as yet. Data mining analyses have revealed that most of the genes encoding enzymes of lipid biosynthesis are represented by gene families reflecting a diversification of isoform functions. The analysis of catalogues of randomly sequenced cDNA seeds reflects the levels of gene expression and provides additional insight as to the regulation of flux 
through the metabolic pathway leading to lipid biosynthesis. The availability of mutant collections and transgenic Arabidopsis lines and the development of microarrays allowing the global analysis of expression of many thousands of genes to be carried out simultaneously will lead to a better understanding of the factors regulating the metabolism of lipids in seeds. Such knowledge will facilitate the manipulation of the fatty acid composition of lipids and the quantity of oils produced in seeds.

Mots-clés : métabolisme lipidique, semences, séquençage systématique, génomique.

Keywords : lipid metabolism, seeds, systematic sequencinq, genomics.

\section{ARTICLE}

La génomique, aboutissement des travaux de biologie moléculaire, peut se comparer à un inventaire. En chimie, les travaux de Mendeleiev ont permis d'effectuer un inventaire exhaustif des éléments chimiques et de prédire leur existence et leur fonction, pour certains d'entre eux. La génomique végétale, qui vise à effectuer, après séquençage total ou partiel du génome, l'inventaire fonctionnel des génomes végétaux, a le même rôle à long terme :

* Elle permet, pour des espèces choisies comme modèle, d'identifier tous les éléments génétiques nécessaires à leur bon fonctionnement. Cet inventaire fait pour un modèle, est valable (contrairement à ce qu'inspire notre intuition) pour toutes les espèces apparentées. Ainsi, l'inventaire effectué pour un génome simple, tel que celui du riz, est utilisable pour toutes les céréales, du fait de la conservation de l'ordre des gènes sur les chromosomes (synténies entre génomes), de même le génome d'Arabidopsis sert de modèle aux légumes, arbres feuillus, oléagineux, etc.

* Elle permet d'assigner un rôle à chacun de ces éléments génétiques, rôle déduit de l'observation des caractéristiques d'une plante chez laquelle la fonction du gène étudié a été abolie (analyse fonctionnelle).

Dans le règne végétal, d'énormes différences de taille entre génomes amènent à privilégier l'inventaire sur des modèles plutôt que sur les espèces cultivées d'importance pour notre économie. Le génome du riz, par exemple, a un contenu d'information similaire environ 40 fois plus petit que celui du blé. De ce fait, la recherche et l'identification de gènes importants chez le blé aura recours aux données accumulées sur l'espèce modèle de référence, le riz, dont le génome sera complètement séquencé (le séquençage du génome est la méthode la plus fiable d'inventaire d'un génome).

\section{Utilisations liées au développement des outils d'analyse des génomes des plantes}

Ces utilisations sont multiples, leur principal avantage résidant dans la possibilité de conduire le travail d'amélioration des plantes non plus de manière aveugle (génétique quantitative) mais de 
manière assistée par l'emploi de marqueurs moléculaires. Pour être fiables, ces marqueurs moléculaires, doivent idéalement s'identifier aux gènes ayant un rôle majeur dans les caractéristiques de la plante et de ses produits récoltés. De plus, l'identification de ces gènes permet à la fois d'en comprendre le rôle et de protéger industriellement leur utilisation par brevet.

Quels gènes représentent des cibles importantes des programmes génomiques ? Ces cibles sont multiples et peuvent être classées en sous-groupes aux intérêts différents :

* Gènes permettant de réduire les intrants et la pollution de l'environnement par les fertilisants

Exemple : efficacité d'utilisation des engrais (nitrate en particulier); gènes de tolérance aux déficits hydriques permettant de réduire les besoins d'irrigation ;

* Gènes de résistance aux maladies (champignons pathogènes, bactéries, nématodes et insectes)

L'introduction de ces gènes par croisements dans les espèces cultivées sera facilitée lorsque ces gènes seront identifiés au niveau moléculaire dans les espèces sauvages où ils se trouvent. Leur introduction dans les plantes cultivées permet de les protéger naturellement et de réduire le recours aux phytosanitaires antifongiques, anti-acariens, insecticides, etc., utilisés souvent massivement en production végétale ;

\section{* Gènes d'architecture et de floraison}

La culture et la récolte des produits végétaux peuvent être grandement facilités par un contrôle approprié de l'architecture et du développement de la plante (nanisme, compétition pour la lumière, précocité de mise à fleur en temps utile, résistance à la verse, inhibition de la formation de graines).

\section{* Gènes de qualité}

Nombreux sont les gènes qui interviennent dans la production de substances indésirables (exemple : substances empêchant la digestibilité, réserves en phosphates dont la forme n'est pas assimilable dans les aliments, substances toxiques telles que les alcaloïdes accumulés par certaines espèces, etc.).

Un nombre croissant de gènes intervenant dans la qualité des produits végétaux sont en cours d'identification (qualité boulangère, synthèse d'acide gras essentiels aux enfants et personnes âgées, protection contre les mécanismes d'oxydation des réserves, qualité des amidons ou des fibres, qualités gustatives des fruits et légumes).

Leur identification moléculaire permettrait d'assurer leur protection industrielle, d'identifier les gènes performants dans ce domaine et de les rassembler dans le génome de plantes élites associé à d'autres caractères agronomiques utiles.

\section{Bénéfices liés à l'acquisition d'un tel savoir-faire}

Les enjeux de l'exploration des génomes de plantes sont cruciaux. De notre investissement dans ce domaine dépend l'autonomie à long terme de la filière semence - production végétale - industrie agroalimentaire de notre pays. Déjà se profile un risque de monopole américain. L'arme agroalimentaire a été maniée par l'Amérique avec efficacité pour assurer son hégémonie après la 
Seconde Guerre mondiale. Nous assistons à une nouvelle phase de recherche d'hégémonie, développée cette fois par des firmes telles que Monsanto ou Pioneer, qui peut compromettre notre autonomie agroalimentaire à long terme.

Par ailleurs, sur le front du développement des nouvelles technologies considérées à juste titre comme sources de création d'entreprises performantes et d'emplois, les technologies d'analyse des génomes ont déjà montré aux États-Unis et, à un moindre degré, en France, leur potentiel créateur d'emplois dans le domaine du génome humain. Investir dans le domaine des génomes végétaux, c'est favoriser, à terme, le développement de sociétés de service en génomique, en relation avec le secteur semencier.

Plus généralement, la nécessité en France d'un programme fédérateur en génomique végétale résulte de l'analyse du contexte de concurrence forcenée entre les groupes des sciences de la vie et de l'agrochimie ayant déjà conduit à une restructuration extrême de l'industrie mondiale des semences, sources de toute filière alimentaire.

\section{Guerre des semences et nécessité d'un programme français de génomique végétale}

\section{Un secteur investi}

Lorsque les premières innovations biotechnologiques trouvent dans les années 1980 leurs applications en amélioration des plantes (haploïdisation, fusions de protoplastes, transgenèse), le secteur des semences apparaît morcelé et principalement aux mains d'entreprises de dimension moyenne, voire familiales et artisanales.

Chacun comprend très vite les potentialités qu'ouvre pour l'amélioration des plantes le premier transfert de gène végétal, réalisé en 1984. Source de toute production agricole, la semence est de plus en plus perçue comme stratégique pour l'alimentation de la planète. Tout au long des années 1990, l'accent donné, notamment en Europe, aux notions de filières et de traçabilité n'a fait que renforcer l'importance de ce secteur, pourtant très limité en taille (marché mondial estimé à 25 milliards de dollars), mais crédité d'un fort potentiel de développement.

À l'inverse, le secteur de l'agrochimie, sensiblement plus significatif en taille (marché mondial de 30 milliards de dollars) apparaît dès les années 1990 fortement concentré et aux mains de grands groupes chimiques et pharmaceutiques. Les innovations biotechnologiques font alors craindre à ce secteur une réduction des intrants chimiques et des transferts d'activité vers l'industrie semencière. Plusieurs de ces groupes prennent donc au long des années 1980 et 1990 des options d'investissements en recherche ou en acquisition de sociétés de biotechnologies en vue de maîtriser l'innovation dans le secteur des semences.

Au-delà de la défense d'un business agrochimique, l'objectif est de capter une valeur sur la chaîne alimentaire, organisée comme indiqué dans la figure 1.

La justification d'un tel bouleversement est donc à rechercher dans l'espoir de maîtriser des filières au travers d'une innovation portant sur l'information biologique, à l'instar de l'industrie des logiciels dans le métier de l'informatique. 
En Europe, l'échec du développement des biotechnologies, à partir de 1998, pénalisera fortement les retours financiers des entreprises ayant mené cette stratégie, mais compte tenu des investissements réalisés (50 milliards de francs pour Dupont et autant pour Monsanto), la concentration du secteur semencier mondial est devenue irréversible. La stratégie de ces groupes sera donc, dans le futur, l'investissement dans des innovations à la fois toujours plus puissantes et mieux acceptées par les différents marchés mondiaux. Les progrès de la connaissance sur les structures des génomes et I'analyse de leurs fonctions offrent des perspectives exponentielles d'application, au service de ces objectifs.

\section{Une propriété intellectuelle en voie de clarification}

Outil de mise à disposition de la connaissance et outil de protection des intérêts de découvreur, le brevet est le système de protection intellectuelle naturel du secteur industriel. Outil spécifique du sélectionneur, le certificat d'obtention végétale (COV) sert à protéger la variété, assemblage de gènes dans un génotype identifié par son phénotype.

Depuis l'origine, les biotechnologies font appel aux brevets et l'on constate depuis quelques années la préférence des professionnels américains pour le brevet variétal, tandis que les sélectionneurs européens confirment leur fidélité au certificat d'obtention végétale, seule protection légale pour une variété sur leur continent.

En génomique, si une tendance mondiale se dessine pour ne pas laisser breveter les séquences génomiques seules, en revanche, un consensus existe pour la protection par brevet de l'invention constituée par la séquence du gène, la démonstration de sa fonction et de son utilisation industrielle.

Il est donc clair aujourd'hui que les connaissances issues de la génomique trouveront des cadres juridiques, parfois partiellement variables selon les continents, mais convergents dans leurs lignes générales. Quels que soient nos souhaits ou nos protestations, ces lignes assureront, dans les filières alimentaires de demain, une maîtrise certaine aux détenteurs des droits de protection intellectuelle sur les grandes espèces végétales.

\section{Une nécessaire réponse française}

La France est l'un des premiers pays agricoles au monde, et le deuxième acteur semencier de la planète. Le rapprochement des deux constats précédents (prise en main du secteur semencier mondial par des groupes chimiques souvent d'origine américaine, et enjeu déterminant de la détention de la propriété intellectuelle) pose, au cours des années 1990, un problème politique à notre pays.

Dans le même temps, chacun des acteurs aujourd'hui réunis dans Génoplante est confronté à des défis auxquels il ne peut, dès 1998, imaginer répondre seul :

* L'Inra et le CNRS sont conscients du risque de leadership américain en génomique végétale dans un contexte de course de vitesse. En effet, une initiative majeure, le National Plant Genome Initiative, doté en 1998 par le Congrès américain de 320 millions de dollars, vient conforter la puissance de feu des groupes industriels outre-Atlantique. Seul le regroupement des compétences françaises et sans 
doute européennes paraît à même d'offrir une alternative et ainsi de pérenniser les importants acquis de la recherche publique française tant en biologie moléculaire végétale qu'en amélioration des plantes.

* Le Cirad et I'IRD sont en outre confrontés aux difficultés des pays du Sud à bénéficier de biotechnologies dont la propriété serait concentrée dans trop peu de mains. Ils souhaitent participer à des programmes dont l'une des retombées serait de faciliter l'accès des pays du Sud aux technologies dans des conditions privilégiées.

* Biogemma (groupes coopératifs semenciers Limagrain et Euralis associés aux filières agricoles céréales, Unigrains, et oléagineuses, Sofiproteol) est créé en 1997 par des agriculteurs autour du concept d'alternative biotechnologique au service des intérêts agricoles français. Une telle structure est par nature en faveur d'un regroupement efficace des compétences nationales en génomique et jouera un rôle moteur dans ce sens.

* Rhône-Poulenc Agrochimie (devenu aujourd'hui Aventis Crop Sciences, ACS), acteur français majeur du secteur, est susceptible d'apporter une compétence biotechnologique et des ressources financières au service du projet. Le fait qu'ACS, contrairement aux autres acteurs internationaux du secteur cités précédemment, ne soit pas intégré dans les semences de grande culture rend possible une collaboration volontariste entre les parties.

* Enfin Bioplante regroupe depuis 1999 deux semenciers céréales français majeurs (Serasem du Groupe Coopératif Sygma et les Établissements Desprez) pour parachever un tour de table très complet des professionnels concernés par les applications des programmes de génomique végétale au secteur de l'agriculture.

Courant 1998, la convergence des analyses de ces acteurs français, souvent d'envergure internationale voire mondiale, est possible car les défis apparaissent clairement à tout observateur souhaitant anticiper la structure de nos secteurs à un horizon de dix ans. De plus, la palette de compétences des différents partenaires tant publics que privés s'avère complète, de l'amont de la biologie moléculaire à la sélection au champ de toutes les espèces de grande culture européennes. Cette palette rend concevable un effort en génomique de niveau mondial.

En revanche, l'idée d'un programme fédérateur se heurte, en 1999, aux habitudes antérieures, aux éventuels a priori des uns sur les autres et à la complexité de tout montage associant autant de compétences dispersées chez des acteurs concurrents. Ceux-ci ont dû apprendre synergies et convergences pour se mettre au service d'un projet partagé, que chacun souhaitait compétitif par rapport à d'autres dispositifs de recherche parfois intégrés dans un même groupe. Aussi, lorsque le programme Génoplante est soumis au ministère de la Recherche, alors dirigé par le ministre Claude Allègre, puis acté le 23 février 1999, si chacun comprend les enjeux, nombreux sont dubitatifs sur notre capacité à organiser une " équipe de France " capable de jouer au plus haut niveau mondial. Cette question trouvera sa réponse dans les résultats du programme. 


\section{Principes organisateurs du programme génoplante}

\section{Objectifs}

Génoplante a pour objectif de développer une recherche en génomique végétale en partenariat public-privé. II a pour mission d'analyser les génomes de plantes cultivées et, en premier lieu, ceux des plantes de grande culture afin d'identifier les bases moléculaires de caractères d'intérêt agronomique. Une large part des efforts porte par ailleurs sur l'analyse fonctionnelle des génomes d'Arabidopsis et du riz qui seront complètement séquencés dans le cadre de collaborations internationales.

Sur la base de ces travaux, Génoplante identifie au niveau moléculaire les gènes importants en production végétale (tolérance aux stress, résistance aux maladies, qualité des produits récoltés et aptitude à la transformation agroalimentaire, etc.) et développe un portefeuille de brevets sur cette base, afin de pouvoir utiliser les allèles performants de ces gènes dans les programmes d'amélioration des plantes. Génoplante n'a pas pour finalité de développer des plantes transgéniques.

Un important effort d'utilisation des outils bio-informatiques est mis en œuvre dans le cadre de ce programme afin d'effectuer l'annotation des séquences génomiques des espèces modèles et de constituer des bases de données conviviales pour les génomes de plantes cultivées. Enfin, des actions sont menées pour étudier le protéome et le réseau métabolique des plantes en liaison avec les travaux de génomique. Ce programme a une base nationale, mais une fois opérationnel, il aura vocation à s'ouvrir à des collaborations, en particulier en ce qui concerne les programmes génériques.

\section{Structure juridique de Génoplante}

Dans un premier temps, la formule du groupement d'intérêt scientifique (GIS) a été mise en place pour gérer Génoplante. La convention constitutive du GIS Génoplante a été approuvée par les partenaires et les pouvoirs publics. Le public et le privé détiennent chacun $50 \%$ des voix et les programmes doivent bénéficier de plus de $50 \%$ des voix pour être décidés. Génoplante doit permettre de générer de la propriété industrielle et de la défendre, et une cellule de valorisation a été constituée à cet effet. Cela implique que Génoplante ait, à terme, une personnalité morale (ce qui n'est pas le cas pour un GIS) et une pérennité à long terme (un brevet a une validité de 20 ans). En complément d'un GIS, une structure de type société par actions simplifiées (SAS), permettant de gérer cette propriété industrielle, a été mise en place. Le développement de propriété industrielle dans le cadre de Génoplante permettra aussi la création de start-up à qui sera confiée l'exploitation d'ensembles de brevets/technologies générés par l'activité de recherche de Génoplante.

\section{Fonctionnement et gestion de la propriété industrielle}

Ceux-ci on été détaillés dans le projet de GIS. La règle générale de financement est de $1 / 3$ ministère, $1 / 3$ organismes publics de recherche, $1 / 3$ privé. Ce financement est établi programme par programme, pour gérer en particulier les droits de propriété qui en résulteront. Les bénéfices qui résulteront de l'activité de Génoplante seront réinvestis dans les programmes de recherche menés par Génoplante et dans la défense des brevets gérés par Génoplante. 
Génoplante est dirigé par un comité stratégique où siègent tous les fondateurs. Un directoire opérationnel est chargé de gérer Génoplante et de mettre en place les programmes préparés par dix comités thématiques appropriés, constitués à parité d'experts du public et du privé (figure 2).

L'évaluation des projets soumis dans le cadre des programmes génériques est faite par des experts internationaux dont les avis sont transmis au comité thématique correspondant. En revanche, les programmes financés majoritairement par l'industrie sont expertisés en interne, par le comité thématique correspondant, pour des raisons de confidentialité. Enfin, un comité scientifique international indépendant et consultatif évalue l'activité de Génoplante.

\section{Programmes génériques}

On regroupe dans ce chapitre les programmes menés sur les génomes modèles, le développement d'outils de génomique et les programmes bio-informatiques de Génoplante. Ces programmes sont financés à $85 \%$ par l'État et à $15 \%$ par les partenaires privés. Cinq comités thématiques ont été constitués.

\section{Analyse fonctionnelle du génome d'Arabidopsis}

Ce programme " post-génomique " vise à identifier la fonction exacte des gènes répertoriés par approche génomique (tri de mutants affectés dans des fonctions importantes, telles que remplissage de la graine, tolérance aux stress abiotiques, résistance aux pathogènes, etc., identification de gènes corégulés et impliqués dans une même fonction par étude d'expression et identification de mutants dans ces gènes).

Les fonctions étudiées sont celles jugées les plus pertinentes d'un point de vue agro-industriel. C'est à ce niveau que peuvent être identifiées le plus rapidement des cibles de protection industrielle recherchées par Génoplante. Les laboratoires concernés sont essentiellement universitaires - CNRS et Inra.

\section{Génomique du riz}

Le riz sert de génome modèle pour les céréales. Le programme riz a donc vocation à servir de ressource pour les programmes menés sur le blé et le maïs. L'essentiel des recherches est développé à Montpellier au Cirad et à I'IRD.

Ce programme est axé sur l'analyse fonctionnelle de ce génome. Il comprend deux volets :

- préparation d'outils nécessaires à l'analyse fonctionnelle du génome du riz (création de mutants d'insertion) ;

- préparation du séquençage du chromosome 12 et séquençage d'une mégabase en 2000. 
Ce programme a pour objectif de développer de nouveaux outils de génomique végétale :

- concevoir des outils d'analyse du transcriptome ;

- produire une collection des lignées ADN-T en environnement contrôlé et identifier des séquences bordures des insertions de cette collection ;

- concevoir ou valider une méthode d'analyse inédite/originale du génome de plantes (exemple : nouvelle méthode de cartographie, de recherche de mutants de délétion) ;

- analyser le protéome.

Ces approches sont développées à l'Unité de recherche en génomique végétale (URGV, Évry) et dans des laboratoires partenaires (CEA, Évry et Grenoble, CNRS, Cirad, Inra, Rhobio).

\section{Bio-informatique}

La bio-informatique de base sur les génomes est soutenue dans un programme séparé pour ses aspects les plus en amont. Les séquences génomiques d'Arabidopsis et du riz doivent être annotées (position des gènes et rôle possible de ceux-ci). Cela nécessite d'adapter les outils existants aux caractéristiques spécifiques des génomes de plantes et, en particulier, de gérer une base de données bibliographiques sur les travaux effectués sur des plantes. Ce programme est développé en réseau (adaptation d'outils aux génomes de plantes tels que l'identification automatique d'introns, de séquences de provenance plastidiale dans le génome nucléaire, de motifs/signaux de régulation, de données bibliographiques, etc.), essentiellement sur les grandes familles multigéniques que comporte le génome d'Arabidopsis. Deux serveurs dédiés à la gestion des données générées par les programmes sont installés à Évry : base privée Rhobio à laquelle se connecte le privé, base publique Génoplante, information localisée à Infobiogen. Divers programmes concernant spécifiquement la gestion des données transcriptome et protéome, les études de proximité entre génomes, l'adressage des protéines dans les plastes, sont développés par ailleurs.

\section{Cibles importantes dans les génomes de plantes cultivées}

Certaines espèces, qui ne justifient pas la création d'un programme de génomique à grande échelle, comportent néanmoins des cibles intéressantes à atteindre du point de vue génomique. Divers projets de clonage positionnel de gènes de résistance aux virus, aux pucerons, ou impliqués dans la mycorrhization sont développés. Par ailleurs, les cartographies de la vigne et du sorgho sont aussi conduites dans ce cadre.

\section{Programmes espèces (blé - maïs - tournesol - pois)}

Les laboratoires publics et privés participent à des recherches sur cinq espèces choisies par Génoplante. Pour chacune d'elles, il existe :

- un programme générique : carte physique dense, banque de grands fragments (BAC - Bacterial Artificial Chromosomes) permettant le clonage positionnel, production et cartographie d'EST (Expressed Sequence Tag) ciblés sur une fonction particulière, données de synténie avec le génome 
modèle de référence (riz pour les monocotylédones et Arabidopsis pour les dicotylédones), analyse bio-informatique des données et analyse du transcriptome dans certains projets. Dans certains cas, un outil de validation " machine à gènes » a été inclus ;

- des programmes ciblés sur des objectifs spécifiques comportant l'identification de Quantitative Trait Locus (QTL), la recherche de gènes candidats pour ces QTL et leur validation. Trois sousprogrammes existent pour chaque espèce :

. un sous-programme « Agronomie » (exemple de la tolérance au stress hydrique chez le maïs) ;

. un sous-programme "Résistance aux maladies " (exemple du clonage d'un gène de résistance à l'Alternaria chez le tournesol) ;

. un sous-programme "Qualité des produits récoltés " (exemple des gènes de valeur boulangère sur le chromosome 5D du blé).

Chaque programme est financé par les partenaires privés et publics selon leurs intérêts respectifs, ce qui déterminera les droits de propriété intellectuelle de ces partenaires, le financeur principal déposant et gérant le brevet. Le plus souvent, les financements sont assurés à $50 \%$ par le public et à $50 \%$ par le privé.

\section{État d'avancement actuel des programmes Génoplante}

\section{Au niveau du financement}

Les fonds nécessaires à la mise en place des programmes proviennent pour la partie publique du Fonds national pour la science (FNS), du Fonds pour la recherche et la technologie (FRT) et du ministère de l'Agriculture et de la Pêche (MAP) et, pour la partie privée, d'Aventis, Biogemma, Bioplante. La notification pour la partie générique au niveau FRT est intervenue le 25 mai 2000, ce qui explique le léger retard pris par ces projets. Le budget acté sur deux ans est de 661 MF. Les contributions sur les deux premières années, hors infrastructures et équipements des laboratoires privés, proviennent à $40 \%$ des organismes publics, $22 \%$ des subventions ministérielles et $38 \%$ du privé.

\section{Au niveau scientifique}

\section{Avancement des projets}

Les programmes scientifiques de Génoplante ont été élaborés, à partir de l'automne 1998, par le biais de comités thématiques paritaires, constitués d'experts du secteur public et privé, qui ont défini les cibles et évalué les projets soumis. Deux grandes catégories de programmes ont été bâties.

Un ensemble de programmes focalisés sur des espèces d'importance agronomique a été mis en place, entre début 1999 et début 2000, par sollicitations des laboratoires sur :

- génomique du maïs 13 projets

- génomique du blé 11 projets 
- génomique du colza 11 projets

- génomique du pois 5 projets

- génomique du tournesol 5 projets

Un ensemble de programmes génériques a été également retenu. Ces projets proviennent d'un appel d'offres, lancé le $1^{\mathrm{er}}$ avril 1999, avec clôture le 15 mai 1999, et évaluation par un panel d'experts internationaux. Sur 88 projets soumis, 41 ont été retenus, certains après modification, qui se répartissent selon les axes suivants :

- analyse fonctionnelle du génome d'Arabidopsis 18 projets

- génomique du riz 1 projet

- bio-informatique 8 projets

- cibles importantes 5 projets

- nouveaux outils 9 projets

Ces projets impliquent trente laboratoires de l'Inra, dix-huit du CNRS, deux du Cirad et un de I'IRD, ainsi que des laboratoires de Biogemma, de Rhobio Évry et de Bioplante. Par ailleurs, quatre laboratoires du CEA et une équipe de I'Institut Pasteur sont également impliqués. Globalement, plus de 300 chercheurs sont impliqués dans ces projets.

Après acceptation par le comité stratégique de Génoplante, les programmes ont démarré :

- blé, maïs mi-1999

- colza janvier 2000

- génériques entre janvier et juin 2000

- pois février 2000

- tournesol mai 2000

Un séminaire réunissant 280 scientifiques impliqués dans Génoplante a été organisé à Montpellier les 25,26 et 27 septembre 2000, afin de partager entre chercheurs l'état d'avancement des différents projets.

Par ailleurs, le premier Conseil scientifique de Génoplante s'est réuni les 4, 5 et 6 octobre 2000 pour faire une première évaluation. Celle-ci s'est révélée très positive, le programme Génoplante étant considéré comme une initiative heureuse et cohérente scientifiquement.

\section{Premiers résultats}

Les programmes Espèces ayant démarré dès 1999, les premiers résultats obtenus concernent la production d'EST de maïs, de blé et de colza, la cartographie de QTL pour ces trois espèces, la construction de banques BAC de colza et de radis). Dans le programme Générique, le programme FST commence à être opérationnel et les premières données peuvent être consultées par les chercheurs 
effectuant des travaux d'analyse fonctionnelle du génome d'Arabidopsis. Six demandes de brevet sont à l'étude et pourraient faire l'objet de dépôt, à l'automne 2000. Par ailleurs, cinq demandes de publications sur des travaux liés à Génoplante ont été examinées et acceptées.

Pour réaliser les programmes, de nombreux recrutements sont effectués ou en cours de mise en place (120 au total, à terme). Ces recrutements ont été répartis entre les partenaires publics et privés. Un certain nombre d'entre eux correspondent à des profils dont l'Inra a besoin à long terme (exemple des bio-informaticiens). D'autres ont été effectués en CDI par les partenaires privés qui souhaitent garder les personnes ainsi recrutées pour leurs activités propres à long terme. Enfin, la formule des CDD classiques, financés sur contrats, a été retenue pour les autres projets.

\section{Implantation d'infrastructures}

La réalisation des programmes a nécessité la mise en place d'infrastructures de recherche. Au niveau privé, un laboratoire Rhobio-génomique a été implanté à Évry. Les travaux d'implantation de ce laboratoire ont été réalisés de juillet 1998 à février 1999 et l'ensemble des équipements nécessaires ( $Q$ bot, équipement de microarrays Amersham) étaient installés en juin 1999. Ce laboratoire joue un rôle central dans les programmes EST de Génoplante et devrait être opérationnel en microarrays durant l'été 2000. Du côté public, trois implantations sont réalisées. Un site de génomique du riz est développé au Cirad. II bénéficie pour ses équipements du soutien de Génoplante et de la Génopole de Montpellier. À Évry, I'Inra a implanté l'unité de recherche en génomique végétale qui a vocation à devenir une unité mixte Inra-CNRS. Cette unité est dirigée par Michel Caboche et est rattachée au département de génétique et d'amélioration des plantes. Les travaux d'implantation ont été effectués par l'Inra, sur fonds propres, de février 1999 à août 1999, les chambres de culture étant livrées en janvier 2000. Les équipements lourds nécessaires à I'URGV sont financés dans le cadre des contrats Génoplante. De ce fait, les fonds nécessaires aux achats d'équipements pour les programmes espèces sont disponibles depuis mars 2000 (achats de robots Biomek et d'un $Q$ bot). Ces équipements seront en place pour la fin de l'année 2000. L'Inra et le ministère de la Recherche ont soutenu la création de I'URGV, en ouvrant des postes de scientifiques, ingénieurs et techniciens dont les recrutements ont été répartis sur les années 1998, 1999 et 2000. Au total, quatre scientifiques, sept ingénieurs et cinq techniciens et administratifs ont été recrutés, en plus de cinq recrutements par mobilité. L'URGV, en plus des programmes Génoplante, aura pour mission de développer des programmes propres de génomique végétale à moyen terme. Par ailleurs, I'Inra a programmé au total, sur la période 2000-2003, le recrutement de seize personnes, ingénieurs et chargés de recherche en génomique végétale.

Enfin, également à Évry, sur le nouveau site Infobiogen, une équipe assurant l'élaboration de la base de données Genoplante-Info, constituée de quatre personnes dont deux ingénieurs Étude Inra et dirigée par Emmanuel Barillot, est en cours de mise en place. À cette occasion, 2,2 MF d'équipements informatiques financés par le FNS sont mis à la disposition du programme Génoplante. La base de données Genoplante-Info a pour vocation, à terme, d'être le support bio-informatique des travaux de génomique végétale à l'Inra et chez ses autres partenaires.

\section{Administration de Génoplante}

Une équipe dédiée au fonctionnement de Génoplante composée de personnes mises à disposition par les membres de Génoplante, est constituée. Elle travaillera en étroite liaison avec le directoire 
opérationnel. Elle doit comprendre, à terme, une dizaine de personnes, ont d'ores et déjà été recrutés : un responsable d'administration et de gestion (Limagrain), un secrétaire général (Inra),une assistante (Inra), un contrôleur de gestion (Biogemma), un webmaster (Biogemma). II reste à recruter deux personnes sur la propriété industrielle, un juriste et un coordinateur scientifique qui doit être identifié.

\section{CONCLUSION}

Au total, une enveloppe a été définie sur cinq ans. Début 2001, un nouvel appel d'offres pour préparer la seconde phase générique doit être lancé, avec sélection des dossiers pour mi-2001. Pour les espèces cultivées, les comités thématiques prépareront la continuation, voire la réorientation des projets, au vu des résultats du Conseil scientifique d'octobre 2000, dès la fin 2000.

Des collaborations internationales sont encouragées. Un séminaire Génoplante/GABI (Genome Analysis in the Biological System of the Plant), organisé par les ministères allemand et français chargés de la Recherche, a eu lieu les 30 et 31 mai 2000, à Bonn. À la suite de ce séminaire, plusieurs types de collaboration sont envisagés :

- des collaborations entre programmes existants : neuf projets prioritaires ont été définis ;

- des rencontres scientifiques : plusieurs rencontres sont prévues, d'ici la fin 2000, sur les thèmes protéomique, analyse fonctionnelle du génome d'Arabidopsis, développement d'outils de mining dans les données bibliographiques, analyse bio-informatique des données EST ;

- la préparation de programmes futurs de collaboration (appel d'offres 2001).

Deux années après son lancement, le programme de génomique végétale français a pris son essor. C'est par la comparaison des résultats obtenus, au terme des cinq premières années, avec ceux des autres grands programmes nationaux de génomique végétale, à l'échelle mondiale, que le premier véritable bilan de cette entreprise pourra être tiré (tableau).

* Ce texte s'appuie sur une présentation du programme Génoplante à l'Académie d'Agriculture, qui a été publiée dans les Comptes rendus de l'Académie d'Agriculture Française (CR Acad Agri Fr 2000 ; 86 (8)). 


\section{Illustrations}

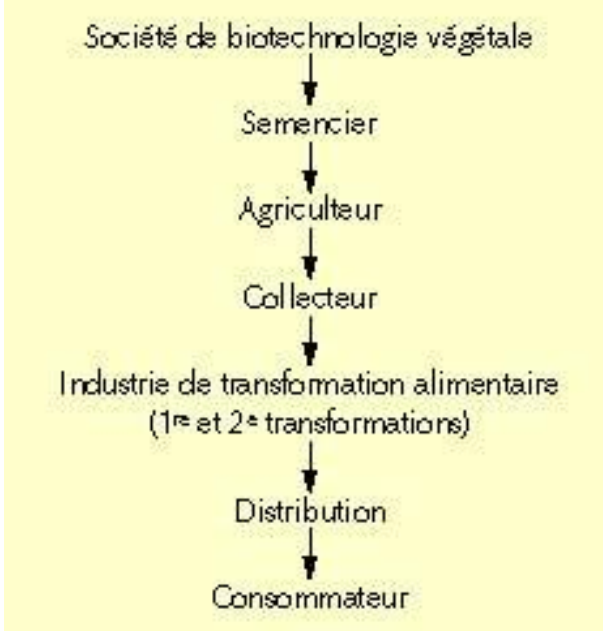

Figure 1. Les acteurs successifs du secteur alimentaire.

Les organes de décision de Génoplante

\begin{tabular}{|c|c|}
\hline Comitế stratég gique & Conseil scientifique \\
\hline Groupe P.l ingénieurs brevets & Directoire \\
\hline \multicolumn{2}{|c|}{ Comitos thématiques paritaires publics-privés } \\
\hline Arabidopsis & Riz \\
\hline Technologies & Bio-informatique \\
\hline Blĕ́ & Mäs \\
\hline Oláoprotáagineux & Rutres espèces \\
\hline
\end{tabular}

Figure 2. Les organes de décision de Génoplante. 
Tableau. Classement des cinq premières entreprises mondiales en semences de gande culture.

\begin{tabular}{|c|c|c|c|}
\hline \multicolumn{2}{|c|}{1995} & \multicolumn{2}{|c|}{1999} \\
\hline Société & Caractéristique & Société & Caractéristique \\
\hline 1. Pioneer & Indépendant & $\begin{array}{l}\text { 1. Pioneer } \\
\text { (Groupe Dupont) }\end{array}$ & Agrochimie \\
\hline 2. Sandoz Seeds & Agrochimie & $\begin{array}{l}\text { 2. Monsanto Seeds } \\
\text { (Dekalb, Cargill, Asgrow) }\end{array}$ & Agrochimie \\
\hline 3. Limagrain & Coopéra tive & $\begin{array}{l}\text { 3. Hille shog NK } \\
\text { (Groupe Syngenta) }\end{array}$ & Agrochimie \\
\hline 4. Dekalb & Indépendant & 4. Limagrain & Coopéra tive \\
\hline 5. Cargill & Indépendant & $\begin{array}{l}\text { 5. Advanta } \\
\text { (Groupe Astra Zeneca) }\end{array}$ & Pha macie \\
\hline
\end{tabular}

